\title{
ЭПИДЕМИОЛОГИЯ ХРОНИЧЕСКИХ РИНОСИНУСИТОВ
}

\author{
Шамкина П.А. ${ }^{1}$, Кривопалов А.А. ${ }^{1}$, Рязанцев С.В. ${ }^{1}$, Шнайдер Н.А. ${ }^{2}$, Гайдуков С.С. ${ }^{1}$, \\ Шарданов 3.H. ${ }^{3}$
}

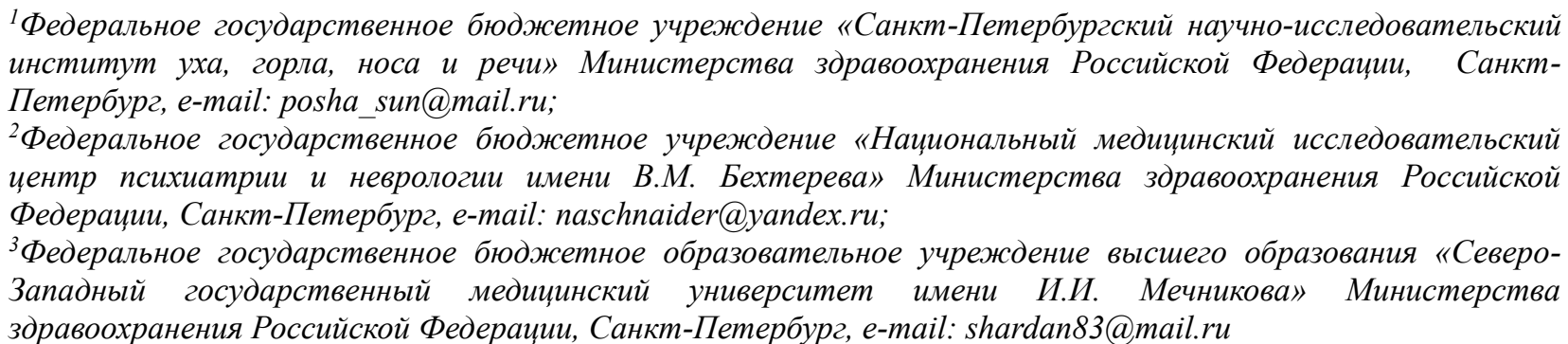

Результаты эпидемиологических исследований распространенности хронического риносинусита в мире и в России неоднозначны. Целью настоящего исследования был поиск и системный анализ публикаций эпидемиологического мониторинга хронического риносинусита в Российской Федерации и в мире. Материалы и методы. Был произведен анализ англо- и русскоязычных публикаций с использованием следующих баз данных: PubMed, MedLine, Web of Science, Russian Science Citation Index, Springer, Scopus, Scientific Research, Google Scholar, Crossref, eLibrary. Глубина поиска составила 45 лет (1974-2019 гг.). В работе представлены данные 62 оригинальных эпидемиологических исследований. Результаты. Показатель распространенности хронического риносинусита варьирует в широком диапазоне в зависимости от региона проживания пациентов, методологического подхода к проведению исследования, возраста пациентов и объема выборки. По данным статистической обработки, средний показатель распространенности хронического риносинусита в мире составил $11,61 \pm 5,47 \%$ разбросом от минимального показателя 1,01\% до максимального показателя 57,6\%. Заключение. В последние годы отмечается рост распространенности хронического риносинусита патологии в различных странах и в Российской Федерации, что требует пересмотра организационно-методического подхода к профилактике, диагностике и лечению хронического риносинусита.

Ключевые слова: хронический синусит, эпидемиология, гиперпластический синусит, хронический риносинусит, распространенность.

\section{EPIDEMIOLOGY OF CHRONIC RHINOSINUSITIS}

\author{
Shamkina P.A. ${ }^{1}$, Krivopalov A.A. ${ }^{1}$, Ryazantsev S.V. ${ }^{1}$, Shnayder N.A. ${ }^{2}$, Gaidukov S.S. ${ }^{1}$, \\ Shardanov Z.N. ${ }^{3}$
}

${ }^{1}$ Federal State Budget-funded Institution "St.-Petersburg Research Institute of Ear, Nose, Throat, and Speech" of the Russian Ministry of Health, Saint-Petersburg, e-mail posha_sun@mail.ru;

${ }^{2}$ Federal State Budget-funded Institution "V.M. Bekhterev National Medical Research Center for Psychiatry and Neurology" of the Russian Ministry of Health, Saint-Petersburg, e-mail nataliashnayder@gmail.com;

${ }^{3}$ Federal State Budget-funded Institution of Higher Education North-Western State Medical University named after I.I. Mechnikova of the Russian Ministry of Health, Saint-Petersburg, e-mail shardan83@mail.ru

The results of the epidemiological studies of the prevalence of chronic rhinosinusitis are ambiguous in the world and in Russia. The purpose of this study was a search and system analysis of the publications of epidemiological monitoring of chronic rhinosinusitis in the Russian Federation and in the world. Materials and methods. The analysis of English- and Russian-language publications was made using the following databases: PubMed, MedLine, Web of Science, Russian Science Citation Index, Springer, Scopus, Scientific Research, Google Scholar, Crossref, eLibrary. Search depth was 45 years (1974-2019). The article presents the results of 62 original epidemiological studies Results. The prevalence rate of chronic rhinosinusitis varies over a wide range depending on the region of patients residence, the methodological approach to the study, the age of the patients and the sample size. According to the statistical processing data, the average prevalence of chronic rhinosinusitis in the world was $11.61 \pm 5.47 \%$ with a spread from the minimum $1.01 \%$ to the maximum $57.6 \%$. Conclusion. In recent years, there has been an increase in the prevalence of chronic rhinosinusitis in various countries and in the Russian Federation, which requires a review of the organizational and methodological approach to the prevention, diagnosis and treatment of chronic rhinosinusitis.

Keywords: chronic sinusitis, epidemiology, hyperplastic sinusitis, chronic rhinosinusitis, prevalence. 
Хронический риносинусит (ХРС) - заболевание, характеризующееся воспалением слизистой оболочки носа и околоносовых пазух, с длительностью симптомов заболевания более 4 недель непрерывно (EP3OS, 2012) [1]. По классификации отечественных авторов, ХРС возникает при длительности заболевания более 3 мес. Информация о распространенности ХРС достаточно сильно варьирует из-за отсутствия общепринятого определения и в зависимости от применяемых диагностических критериев: учета клинических проявлений заболевания, результатов лучевых методов исследования или диагностической пункции околоносовых пазух [2].

Цель исследования: поиск и системный анализ публикаций эпидемиологического мониторинга хронического риносинусита в Российской Федерации и в мире.

Материалы и методы исследования. Был произведен анализ англо- и русскоязычных публикаций с использованием следующих баз данных: PubMed, MedLine, Web of Science, Russian Science Citation Index, Springer, Scopus, Scientific Research, Google Scholar, Crossref, eLibrary. Глубина поиска 1974-2019 гг. (45 лет). Поиск осуществлялся по следующим ключевым словам на русском и английском языках: хронический синусит, эпидемиология, гиперпластический синусит, хронический риносинусит, распространенность, chronic sinusitis, epidemiology, hyperplastic sinusitis, chronic rhinosinusitis, prevalence. Согласно критериям поиска найдено 48988 статей. Однако цели настоящего исследования соответствовало 4930 работ, среди которых в базах было найдено: PubMed (2420 статей), Web of Science (1540), Scopus (1230), Google Scholar (1900), Crossref (1725), eLibrary (25). Из них проанализировано 62 полнотекстовые статьи на русском и английском языках.

\section{Результаты исследования}

Проведенный поиск показал, что число оригинальных исследований, посвященных вопросу эпидемиологии ХРС, ограниченно. Использовались различные методологические подходы к диагностике ХРС и различные дизайны исследования, что в определенной мере затрудняло проводимый метаанализ.

\section{Российская Федераџия}

По данным Ю.К. Янова и соавт. (2002), в России синуситом страдают около 10 млн человек в год, а среди пациентов, госпитализированных в ЛОР-стационары, данная патология составляет от 15 до 36\% [3]. А.С. Лопатин и В.П. Гамов (2011) предоставили информацию об увеличении распространенности ХРС в 2 раза за последние 20 лет. Имеется корреляция между распространенностью ХРС и возрастом пациентов, с увеличением 
возрастного порога увеличивается и распространенность: от 20 до 29 лет она составляет 2,7\%, от 50 до 59 лет $-6,6 \%$ [4].

По данным Бюро медицинской статистики Департамента здравоохранения Москвы на 2012 год, распространенность синуситов составляла 1420 случаев на 100000 взрослого населения (1,42\%) [5]. Согласно данным А.Б. Туровского (2013), синусит занимает первое место среди всех хронических заболеваний в оториноларингологии (146/1000 населения, т. е. $14,6 \%$ ). В среднем около 5-15\% взрослого населения и 5\% детей страдают той или иной формой синусита. За последние 10 лет отмечается рост заболеваемости синуситами в 3 раза, а больные, госпитализированные по поводу болезней околоносовых пазух, составляют примерно $2 / 3$ от общего числа пациентов специализированных стационаров [6]. По результатам московского исследования О.А. Иванченко и А.С. Лопатина (2013), среди опрошенных пациентов симптомы ХРС отмечали 35\% респондентов [7]. По данным В.Т. Пальчуна (2015), за период с 2009 по 2012 г. в Москве на амбулаторном приеме ЛОРспециалистов доля пациентов, страдающих ХРС, уменьшилась с 12,0 до 11,0\% [8].

А.А. Кривопалов и соавт. (2015) представили результаты крупного эпидемиологического исследования распространенности заболеваний ЛОР-органов в 32 субъектах РФ, в структуре которого воспалительные заболевания носа и околоносовых пазух составили 32,31 $\pm 2,02 \%$ [9]. По данным А.И. Крюкова и соавт. (2017), в период с 1998 по 2015 г. в поликлиниках Москвы число пациентов, обратившихся по поводу ХРС, было стабильным и составило $0,74 \%$ от общего числа пациентов с патологией ЛОР-органов в год. Отмечалось увеличение числа больных с ХРС за период с 2012 по 2015 г., которое достигло уровня 52112 пациентов, что составило 3,17\% от общего числа больных, пролеченных в поликлиниках Москвы. В период с 2009 по 2015 г. число больных, госпитализированных с ХРС, уменьшилось на 23,8\% и достигло уровня 2816 пациентов $(8,3 \%$ от общего числа больных ЛОР-стационаров) [10].

Северная Америка

По данным Национального опроса общественного здравоохранения (The National Health Interview Survey), проведенного в 1996 году, ХРС является вторым по распространенности хроническим состоянием здоровья, поражает 12,5\% населения США, что составляет приблизительно 31 миллион пациентов в год [11]. Согласно данным J.С. Collins (1997), распространенность ХРС составила 15,5\% от общей численности населения США, причем уровень заболеваемости ХРС у женщин была выше, чем у мужчин, в соотношении 6/4 [12].

Национальный опрос общественного здравоохранения (1997) представил данные о распространенности ХРС, что составило 16,3\%. Среди всех опрошенных пациентов 
большую долю занимали лица женского пола (20,6\% и $11,5 \%$ соответственно). В возрастной группе 45-64 года диагноз ХРС ставился чаще (19,3\%), чем в группах 18-44 года (15,3\%), 65 74 года $(15,6 \%)$ и старше 75 лет $(13,2 \%)$. Процент взрослых пациентов с ХРС отмечался более высоким в южных регионах страны (19,5\%) по сравнению с другими регионами США (ниже 15,7\%) [11].

По данным R.G. Shashy и соавт. (2004), распространенность ХРС составила 2\% населения в Олмстеде (штат Миннесота, США) [13]. Анализ данных Национального опроса здоровья в США (2008) показал, что ХРС поражает в среднем каждого 7-го взрослого. Среди всех опрошенных 13,6\% (30621 пациент) сообщили о диагностированном у них ранее ХРС. Частота диагностированного ХРС у женщин была выше (17,4\%), чем у мужчин $(9,25 \%)$. Также был произведен анализ распределения ХРС в расовой/этнической популяции. ХРС регистрировался реже среди азиатов (7\%) в сравнении с темнокожими $(13,8 \%)$ и белыми респондентами $(13,8 \%)$. Также отмечалась более низкая распространенность ХРС среди латиноамериканцев $(9,3 \%)$ в отличие от неиспаноязычных белых $(14,7 \%)$ и темнокожих американцев $(13,9 \%)$ [14].

По данным Национального обследования состояния здоровья (2012), которое проводится каждые 6 лет среди взрослого населения в США, была выявлена тенденция к снижению распространенности ХРС с 16,3\% в 1997 году до 14\% в 2006 году и 12,1\% в 2012 году $[11 ; 15]$. N. Bhattacharyуа (2012) представил данные о числе больных ХРС в США: 11,1 $\pm 0,48$ миллиона взрослых пациентов сообщили об установленном диагнозе ХРС в 2007 году, что составило 4,9 \pm 0,2\% населения США [16]. Согласно обзору С. Bachert и соавт. (2015), ХРС в настоящее время признан заболеванием, поражающим около $10 \%$ взрослого населения в промышленно развитых странах [17]. A.G. Hirsch и соавт. (2016) представили результаты исследования распространенности ХРС в штате Пенсильвания (США), что составило 11,9\%. Максимальная распространенность ХРС была в возрасте от 50 до 59 лет (15,9\%), минимальная - в возрасте после 69 лет (6,8\%) [18]. По результатам Национального обследования здоровья, представленным N. Bhattacharyya и S. Gilani (2018), 2,1\% взрослого населения США страдают ХРС [19]. По данным исследования Ү. Chen и соавт. (2003), проведенного в Канаде, распространенность ХРС варьировала от 3,4\% у мужчин до 5,7\% у женщин. Распространенность ХРС увеличивалась с возрастом, составляя в среднем 2,7\% и 6,6\% в возрастных группах 20-29 и 50-59 лет соответственно. После 60 лет уровень распространенности ХРС уменьшался до 4,7\% [20]. Ү. Хи и соавт. (2016) представили данные исследования распространенности ХРС в Канаде, которые составили 2,1\% (в диапазоне от 1,9 до 2,3\% в зависимости от возраста) [21]. 
S.F. Ahsan и соавт. (2004) представили информацию о распространенности ХРС в Республике Тринидад и Тобаго (государство на территории Карибского бассейна), составившей 9,3\% [22]. По результатам R.R. Pilan и соавт. (2012), распространенность ХРС в Сан-Паулу (Бразилия) составила 5,5\%. Данные были получены на основании индивидуальных опросов и применении критериев EPOS (2012) [23].

Eвpona

F. Gordts и соавт. (1996) предоставили данные об оценке частых ХРС среди 99 пациентов из Бельгии, проходивших магнитно-резонансное исследование (МРТ) по подозрению на внутричерепное заболевание, по результатам которого более чем в 40\% случаев была выявлена патология околоносовых пазух. По данным анкетирования пациентов, были отмечены следующие жалобы: выделения из носа (6\%), двусторонняя обструкция носа (19\%) [24]. По данным S.F. Ahsan и соавт. (2004), распространенность ХРС в Абердине (Шотландия) составило 9,6\%. В ходе этого исследования проводился сравнительный анализ распространенности ХРС в умеренном (Шотландия) и тропическом (Карибское море) климатических поясах. Распространенность ХРС оказалась сопоставимой (9,6\% и 9,3\% соответственно), на основании чего был сделан вывод об одинаковой частоте встречаемости ХРС в обеих климатических зонах [22]. А.Р. Сакович (2009) представил результаты эпидемиологического исследования ЛОР-патологии в Белоруссии в период 20012008 гг. Среди всех госпитализированных было определено 4140 (19,32\%) пациентов с ХРС. В 2003 г. был отмечен рост распространенности ХРС до 22,2\% и стабилизации показателей в 2005-2008 гг. $(20,7-22,6 \%)[25]$.

Согласно исследованию Европейской сети по глобальной аллергии и астме (GA2LEN, 2011), общая распространенность ХРС по критериям EP3OS составила 10,9\% (95\% ДИ: 6,927,1\%). Отмечены значительные различия в распространенности ХРС. В изученных центрах среднее значение распространенности было определено в Лондоне (Великобритания) и составило 10,0\% (95\% ДИ: 8,5-11,7\%). Самая высокая распространенность была в Коимбре (Португалия) (27,1\%; 95\% ДИ: 25,0-29,3\%), причем нижний предел 95\% ДИ значительно превышал оценки других центров. Три центра в Польше $(14,4 \%, 17,3 \%, 19,7 \%)$, Амстердаме (Нидерланды) (14,3\%), Генте (Бельгия) (18,8\%), Дуйсбурге (Германия) (14,1\%) и Монпелье (Франция) $(13,3 \%)$ имели показатели распространенности значительно выше среднего значения. Все центры в Скандинавии, за исключением Стокгольма (Швеция) (9,6\%), центры в Бранденбурге (Германия) (6,9\%) и Скопье (Македония) $(8,2 \%)$ имели показатели распространенности значительно ниже среднего значения. Наиболее контрастный показатель распространенности ХРС внутри страны был отмечен в Германии, где распространенность составила $6,9 \%$ в Бранденбурге и $14,1 \%$ в Дуйсбурге [26]. В 
исследовании GA2LEN у 61,7\% (95\% ДИ: 50,3-72,3\%) лиц с EP3OS-признаками ХРC диагноз подтверждался результатами эндоскопии. И, напротив, при эндоскопическом исследовании признаки ХРС выявлены у 38\% (95\% ДИ: 32,3-44,1\%) лиц, не имевших субъективных симптомов заболевания. Среди пациентов с выявляемой симптоматикой и без симптомов заболевания диагноз ХРС был установлен в $31,4 \%$ и $11,1 \%$ случаях соответственно [27].

T. Thilsing и соавт. (2012) представили результаты эпидемиологического исследования ХРС в Дании, показавшего распространенность ХРС 7,8\% без значимых различий по возрасту и полу пациентов [28]. По данным исследования R. Hoffmans и соавт. (2018), проведенного в Нидерландах, распространенность ХРС составила $16 \%$, с вариацией от 13\% до $18 \%$ в разных областях страны [29].

\section{Южная и Юго-Восточная Азия}

В период с июля по октябрь 1991 г. в Корее было проведено Национальное исследование распространенности и факторов риска ХРС. Распространенность ХРС составила $1,01 \%$, в том числе: $1,07 \%$ - у мужчин и $0,96 \%$ - у женщин. По анализу географического расположения была выявлена более высокая распространенность ХРС в прибрежных городах Кореи, таких как Пусан $(1,67 \%)$ и Инчон (3,09\%) [30]. В 2008 г. в Корее был проведено Национальное обследование здоровья и питания (KNHANES) в 18 провинциях страны. Распространенность ХРС составила 6,95\%, в том числе: у мужчин 8,24\%, у женщин - 6,0\%. Среди пациентов в возрасте от 19 до 29 лет были отмечены самые низкие эпидемиологические показатели (3,95\%), тогда как в возрасте пациентов 70 лет определен самый высокий показатель распространенности ХРС (10,16\%) [31]. Согласно данным исследования Y.S. Сhо и соавт. (2010), распространенность ХРС в Корее составила $7,12 \%$, с большей долей среди мужского населения (8,16\% у мужчин против 6,10\% у женщин). Максимальная распространенность ХРС отмечалась в возрасте пациентов старше 60 лет (10,5\%), минимальная распространенность была в возрастной группе 19-29 лет (4,34\%) [32]. J.-С. Ahn и соавт. (2016) представили данные 5-летнего Национального обследования здоровья и питания в Корее (KNHANES, 2008-2012). Распространенность ХРС у взрослых составила 8,4\% с преобладанием среди лиц мужского пола $(9,6 \%$ у мужчин против 7,1\% у женщин). С увеличением возраста распространенность ХРС увеличивалась с достижением максимального показателя $(10,2 \%)$ в возрасте старше 70 лет, минимальная распространенность ХРС отмечалась в возрастной период от 20-49 лет (7,5-7,6\%) [33]. По данным J.Н. Кim и соавт. (2016), распространенность ХРС в Корее составила 10,78\% на основе клинических симптомов, а по результатам эндоскопического исследования - 1,20\% [34]. Согласно результатам исследования C.S. Hwang и соавт. (2018), распространенность 
ХРС в Корее составила 5,88\%. Среди пациентов старше 65 лет распространенность ХРС была выше (6,55\%) по сравнению с пациентами возрастной группы 19-64 года (5,69\%) [35].

По результатам J.В. Shi и соавт. (2015), распространенность ХРС в Китае составила 8,0\% (95\% ДИ: 4,8-9,7\%). Распространенность ХРС была выше среди мужчин (8,79\%), чем среди женщин (7,28\%) [36]. Согласно данным исследования Q.-L. Fu и соавт. (2015), распространенность ХРС в Гуанчжоу (Китай) составила 8,4\% [37]. По данным X.D. Wang и соавт. (2016), распространенность ХРС в Китае в общей популяции составила 2,1\%. После поправки на возрастное распределение популяций медиана распространенности ХРС составила 2,2\% [38]. W.-X. Gao и соавт. (2016) представили результаты эпидемиологического исследования в Китае, согласно которому распространенность ХРС составила 7,99\%. Среди лиц мужского пола распространенность ХРС была выше (52,9\% у мужчин против 47,1\% у женщин). Максимальная распространенность ХРС определялась в возрастной группе от 3559 лет (45,7\%) в сравнении с 4,8\% в группе 0-14 лет, 33,0\% - в группе 15-34 и 18,8\% - в группе старше 60 лет [39].

B.R.V. Varma и G.V. Kumar (2017) представили данные исследования, проведенного в штате Телангана (Индия). Среди обследованных пациентов ХРС был выявлен в 57,6\% случаев. Среди пациентов женского пола распространенность была несколько выше (60\% у женщин против 56,3\% у мужчин) [40] (таблица 1).

Таблица 1

\section{Распространенность хронического риносинусита в мире}

(Шамкина П.А. и соавт., 2019 г.)

\begin{tabular}{|c|c|c|c|}
\hline Автор, год & Страна (регион) & $\mid \begin{array}{l}\text { Распространенность } \\
\text { хронического } \\
\text { риносинусита, \% }\end{array}$ & Ссылка \\
\hline \multicolumn{4}{|c|}{ РФ } \\
\hline Янов Ю.К., 2002 & Россия & 25,5 & {$[3]$} \\
\hline Лопатин А.С., 2011 & Россия & 4,65 & {$[4]$} \\
\hline Крюков А.И. 2012 & Россия, Москва & $\mid 1,42$ & {$[5]$} \\
\hline Туровский А.Б., 2013 & Россия & $\mid 14,6$ & {$[6]$} \\
\hline Иванченко О.А., 2013 & Россия, Москва & 35,0 & {$[7]$} \\
\hline Пальчун В.Т., 2015 & Россия, Москва & 11,5 & {$[8]$} \\
\hline Кривопалов А.А., 2015 & Россия & 32,31 & {$[9]$} \\
\hline Крюков А.И., 2017 & Россия, Москва & 32,31 & {$[10]$} \\
\hline \multicolumn{4}{|c|}{ Северная Америка } \\
\hline
\end{tabular}




\begin{tabular}{|c|c|c|c|}
\hline Collins J.G., 1997 & США & 15,5 & [12] \\
\hline Adams P.F., 1999 & США & 12,5 & [11] \\
\hline Blackwell D.L., 2002 & США & 16,3 & {$[11]$} \\
\hline Shashy R.G., 2004 & США, Олмстед, Миннесота & 2,0 & [13] \\
\hline Pleis J.R., 2007 & США & $\mid 14,0$ & {$[15]$} \\
\hline Pleis J.R., 2009 & США & $\mid 13,6$ & [15] \\
\hline Bhattacharyya N., 2011 & США & 4,9 & [16] \\
\hline Soler Z.M., 2012 & США, афроамериканцы & 13,8 & {$[14]$} \\
\hline Soler Z.M., 2012 & США, латиноамериканцы & 8,8 & [14] \\
\hline Soler Z.M., 2012 & США, европейцы & $\mid 13,0$ & [14] \\
\hline Soler Z.M., 2012 & США, азиаты & 7,0 & [14] \\
\hline Blackwell D.L., 2014 & США & $\mid 12,1$ & $\mid[15]$ \\
\hline Bachert C., 2015 & США, Европа & $\mid 10,0$ & $\mid[17]$ \\
\hline Hirsch A.G., 2016 & США, Пенсильвания & 11,9 & [18] \\
\hline Bhattacharyya N., 2018 & США & 2,1 & [19] \\
\hline Chen Y., 2003 & Канада & 4,55 & {$[20]$} \\
\hline Xu Y., 2016 & Канада & 2,1 & [21] \\
\hline \multicolumn{4}{|c|}{ Южная Америка } \\
\hline Ahsan S.F., 2004 & $\begin{array}{l}\text { Карибские острова, } \\
\text { Тринидад и Тобаго }\end{array}$ & 9,3 & [22] \\
\hline Pilan R.R., 2012 & Бразилия, Сан-Паулу & 5,5 & [23] \\
\hline \multicolumn{4}{|c|}{ Европа } \\
\hline Gordts F., 1996 & Бельгия & 6,0 & [24] \\
\hline Ahsan S.F., 2004 & Шотландия & 9,6 & {$[22]$} \\
\hline Сакович А.Р., 2009 & Белоруссия, Минск & 19,32 & {$[25]$} \\
\hline Tomassen P., 2010 & Европа & 21,25 & {$[27]$} \\
\hline $\begin{array}{l}\text { Hastan D., } 2011 \\
\text { GA2LEN study }\end{array}$ & Европа & 10,9 & [26] \\
\hline Hastan D., 2011 & Швеция, Гётеборг & 8,3 & {$[26]$} \\
\hline Hastan D., 2011 & |Швеция, Стокгольм & 9,6 & [26] \\
\hline Hastan D., 2011 & Швеция, Умео & 8,1 & [26] \\
\hline
\end{tabular}




\begin{tabular}{|c|c|c|c|}
\hline Hastan D., 2011 & Швеция, Уппсала & 8,6 & {$[26]$} \\
\hline Hastan D., 2011 & Финляндия, Хельсинки & 6,9 & {$[26]$} \\
\hline Hastan D., 2011 & Дания, Оденсе & 7,9 & {$[26]$} \\
\hline Hastan D., 2011 & Польша, Катовице & 17,3 & {$[26]$} \\
\hline Hastan D., 2011 & Польша, Краков & 19,7 & {$[26]$} \\
\hline Hastan D., 2011 & Польша, Лодзь & 14,4 & {$[26]$} \\
\hline Hastan D., 2011 & Великобритания, Лондон & 10,0 & {$[26]$} \\
\hline Hastan D., 2011 & Великобритания, Саутгемптон & $\mid 11,2$ & {$[26]$} \\
\hline Hastan D., 2011 & Нидерланды, Амстердам & 14,3 & {$[26]$} \\
\hline Hastan D., 2011 & Бельгия, Гент & 18,8 & {$[26]$} \\
\hline Hastan D., 2011 & Германия, Бранденбург & 6,9 & {$[26]$} \\
\hline Hastan D., 2011 & Германия, Дуйсбург & 14,1 & {$[26]$} \\
\hline Hastan D., 2011 & Франция, Монпелье & 13,3 & {$[26]$} \\
\hline Hastan D., 2011 & Македония, Скопье & 8,2 & {$[26]$} \\
\hline Hastan D., 2011 & Италия, Палермо & 10,9 & {$[26]$} \\
\hline Hastan D., 2011 & Португалия, Коимбра & 27,1 & {$[26]$} \\
\hline Thilsing T., 2012 & Дания & 7,8 & {$[28]$} \\
\hline Hoffmans R., 2018 & Нидерланды & 16,0 & [29] \\
\hline \multicolumn{4}{|c|}{ Южная и Юго-Восточная Азия } \\
\hline Min Y.G., 1996 & Южная Корея & 1,01 & {$[30]$} \\
\hline Cho Y.S., 2010 & Южная Корея & 7,12 & {$[31]$} \\
\hline Kim, Y. S., 2011 & Южная Корея & 6,95 & {$[32]$} \\
\hline Ahn J.-C. , 2016 & Южная Корея & 8,4 & {$[33]$} \\
\hline Kim J.H., 2016 & Южная Корея & 10,78 & {$[34]$} \\
\hline Hwang C.S., 2018 & Южная Корея & 5,88 & {$[35]$} \\
\hline Shi J.B., 2015 & Китай & 8,0 & {$[36]$} \\
\hline Fu Q.-L, 2015 & Китай, Гуанчжоу & 8,4 & {$[37]$} \\
\hline Wang X.D., 2016 & Китай & 2,15 & {$[38]$} \\
\hline Gao W.-X., 2016 & Китай & 7,99 & [39] \\
\hline Varma B.R.V., 2017 & Индия & 57,6 & {$[40]$} \\
\hline
\end{tabular}


Также были изучены доступные публикации, посвященные распространенности в мире ХРС с полипами в носу или околоносовых пазухах. По данным исследования С.Ј. Falliers (1974), распространенность ХРС с полипами в США составила 0.2\% [41]. А по данным исследования G.A. Settipane и F.H. Chafee (1977), распространенность ХРС с полипами в США составила 4,2\%, с максимальным показателем у пациентов с бронхиальной астмой $(6,7 \%)$ [1]. Д.И. Тарасов и А.Б. Морозов (1991) показали, что в России ХРС с полипами встречается у 12-13\% пациентов, наблюдаемых амбулаторно у ЛОР-врачей, и у 2030\% больных ЛОР-отделений стационаров [42]. P.L. Larsen и M. Tos (1991) представили результаты исследования кадаверного материала в Копенгагене (Дания), согласно которым распространенность ХРС с полипами составила 2\% [1]. A. Drake-Lee (1993) показал, что распространенность ХРС с полипами в популяции Англии варьирует в диапазоне 0,2-1\% [1]. По результатам исследования, представленного А. Davidsson и H.B. Hellqiust (1993), распространенность ХРС с полипами в Эребру (Швеция) составила 0,04\% [43]. Согласно результатам W. Hosemann и соавт. (1994), распространенность ХРС с полипами в Германии составила $1,5 \%$ [1].

Согласно исследованию Г.М. Портенко (1995), в России распространенность ХРС с полипами составила 1,3-5,5\% [44]. А.А. Ланцов и соавт. (1999) представили данные о распространенности ХРС с полипами в Санкт-Петербурге, которая составила 0,05\% (от 1,3 до 13,1 на 10000 населения в разных поликлиниках города) [45]. По данным исследования C. Bachert и соавт. (1999), распространенность ХРС с полипами в Германии составила 4\% [1]. J. Hedman (1999) представил данные о распространенности ХРС с полипами в Финляндии, составившей 4,3\% среди взрослого населения страны [1]. Согласно данным К. Larsen и M. Tos (2002), распространенность ХРС с полипами в Рибе (Дания) составила 0,06\%, показатель был выше среди мужского населения (0,086\% у мужчин против 0,039\% у женщин) [1].

По данным А.С. Лопатина (2003), ХРС с полипами был обнаружен у 1,02\% пациентов, проживающих в Москве [46]. По результатам исследования L. Johansson и соавт. (2003), проведенного в Шёвде (Швеция), распространенность ХРС с полипами составила 2,7\%. Среди мужчин распространенность была выше, чем среди женщин $(2,2 \%$ и 1,0\% соответственно). Максимальный показатель распространенности был отмечен в возрасте 60 лет $(5,0 \%)$ [1]. Согласно исследованию J.M. Klossek и соавт. (2005), проведенному во Франции, распространенность ХРС с полипами составила 2,1\% [1]. По данным Y.S. Cho и соавт. (2010), распространенность ХРС с полипами в Корее составила 2,53\%, без различий по полу. Максимальная распространенность была в возрасте 60 лет и старше (5,36\%) [47]. J. We и соавт. (2015) показали, что в Корее распространенность ХРС с назальными полипами 
составила 2,5\%: у мужчин - 3,2\%, у женщин - 1,9\% [48]. J.B. Shi и соавт. (2015) представили результаты исследования распространенности ХРС с полипами в Китае, которая составила 1,1\% [36]. По результатам Национального обследования здоровья и питания в Корее, опубликованного Ј.-С. Аhn и соавт. (2016), распространенность ХРС с полипами составила 2,6\% и без полипов - 5,8\% [33]. По данным B.R.V. Varma и G.V. Kumar (2017), среди обследованных пациентов в штате Телангана (Индия) была высокой распространенность ХРС с полипами и составила 42,4\%. Среди мужчин ХРС с полипами определялся у 43,7\%, среди женщин - у 40\% [40].

\section{Обсуждение результатов}

Таким образом, проведенный нами критический анализ доступных публикаций, отражающих эпидемиологические исследования ХРС в различных странах мира, свидетельствует о том, что показатель распространенности ХРС варьирует в широком диапазоне в зависимости от региона проживания пациентов (рисунок), методологического подхода к проведению исследования (анкетирование, национальный регистр, госпитальный регистр, эндоскопический скрининг, МРТ-скрининг пациентов, имеющих или не имеющих специфические для ХРС жалобы и клиническую симптоматику), возраста пациентов и объема выборки.

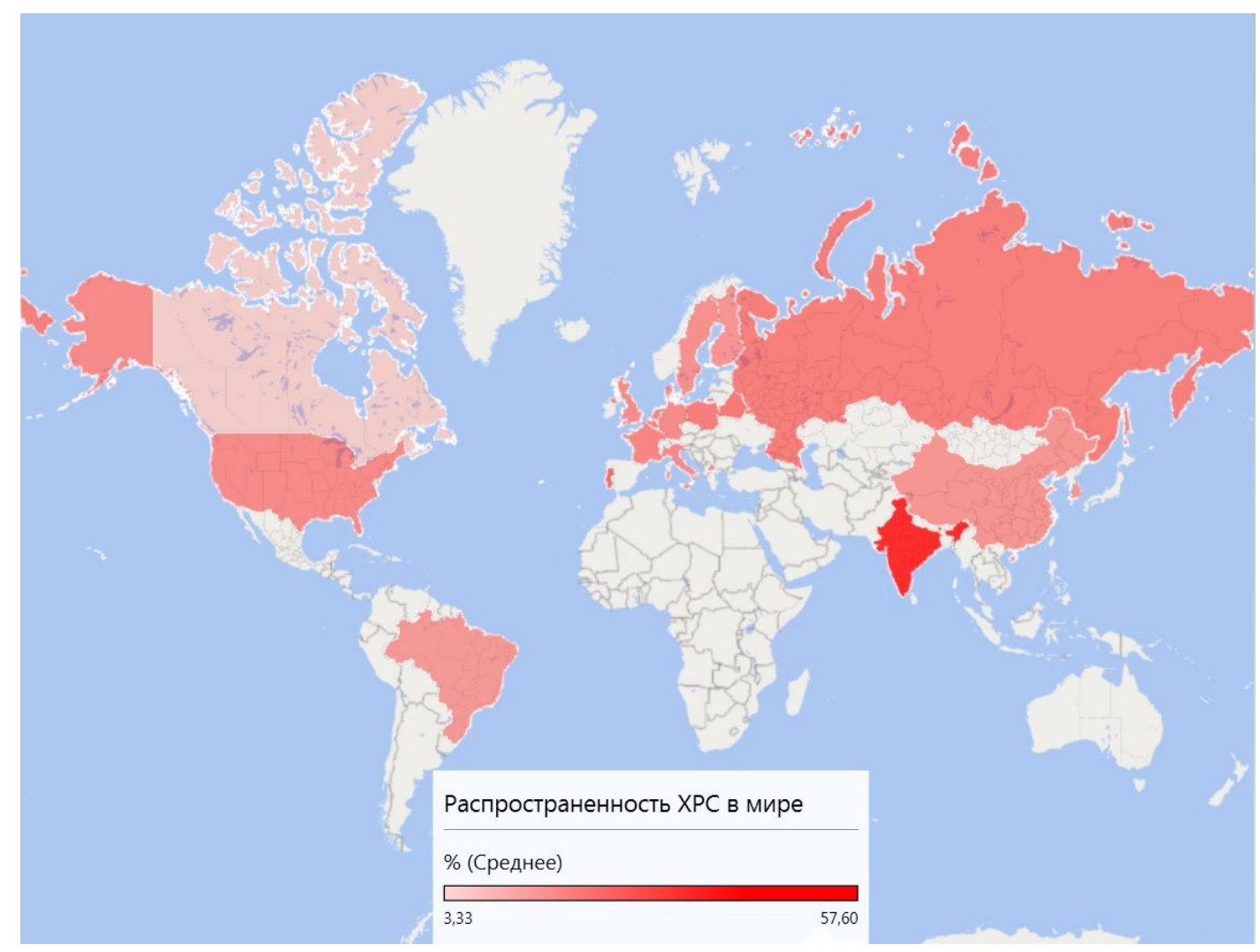

Распространенность хронического риносинусита в мире (Шамкина П.А. и соавт., 2019 г.)

По результатам статистической обработки полученных нами данных, средний 
показатель распространенности ХРС в мире составил 11,81 \pm 5,81\% (95\% ДИ: 6,0-17,62\%) с разбросом от минимального показателя 1,01\% (Южная Корея), до максимального показателя $57,6 \%$ (Индия).

Анализируя эпидемиологические показатели в различных регионах мира, нами показано, что распространенность ХРС в РФ колебалась от 1,42 до 35\%, средний показатель $16,42 \pm 10,89 \%$ (95\% ДИ: 5,53-27,31\%). В странах Северной Америки средний показатель распространенности ХРС составил 9,66 +4,25\% (95\% ДИ: 5,41-13,91\%), с вариацией от 2 до 16,3\%. В странах Южной Америки средний показатель распространенности ХРС колебался от 5,5 до 9,3\%, со средним показателем 7,4 \pm 1,9\% (95\% ДИ: 5,5-9,3\%). Распространенность ХРС в Европе варьировала от 6 до 27,1\%, средний показатель равен 12,56 \pm 4,42\% (95\% ДИ: 8,14-16,98\%). В странах Юго-Восточной Азии распространенность ХРС колебалась от 1,01 до 57,6\%, средний показатель распространенности ХРС (за исключением максимального показателя в Индии) составил 6,67 $\pm 2,19 \%$ (95\% ДИ: 4,48-8,86\%).

В большей части проанализированных нами исследований не проводилось разделение пациентов на группы в зависимости от характера патологического процесса в околоносовых пазухах при ХРС. Однако было найдено 20 работ, в которых проводилось эпидемиологическое исследование ХРС с полипами в носу или околоносовых пазухах (таблица 2). Частота распространенности ХРС с полипами варьировала от минимального 0,04\% (Швеция) до максимального 42,4\% (Индия). Средний показатель распространенности

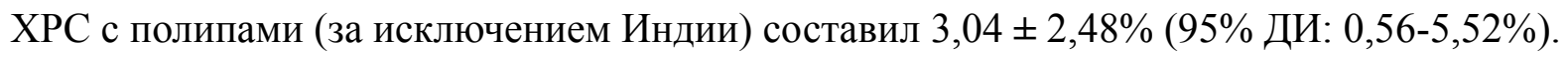

Таблица 2

Распространенность хронического риносинусита с полипами в мире

(Шамкина П.А. и соавт., 2019 г.)

\begin{tabular}{|c|c|c|c|}
\hline Автор, год & Страна (регион) & $\begin{array}{l}\text { Распространенность } \\
\text { хронического риносинусита, } \\
\%\end{array}$ & Ссылка \\
\hline Falliers C.J., 1974 & США & 0,2 & {$[41]$} \\
\hline Settipane G.A., 1977 & США & 7,55 & {$[1]$} \\
\hline Тарасов Д.И., 1991 & Россия & 19,5 & {$[42]$} \\
\hline Larsen P.L., 1991 & Дания, Копенгаген & 2,0 & {$[1]$} \\
\hline Drake-Lee A., 1993 & Англия & 0,6 & {$[1]$} \\
\hline Davidsson A., 1993 & Швеция, Эребру & 0,04 & {$[43]$} \\
\hline Hosemann W., 1994 & Германия & 1,5 & {$[1]$} \\
\hline Портенко Г.М., 1995 & Россия & 3,4 & {$[44]$} \\
\hline Ланцов А.А., 1999 & Россия, Санкт-Петербург & 0,05 & {$[45]$} \\
\hline
\end{tabular}




\begin{tabular}{|l|l|l|l|} 
Bachert C., 1999 & Германия & 4,0 & {$[1]$} \\
\hline Hedman J., 1999 & Финляндия & 4,3 & {$[1]$} \\
\hline Larsen K., 2002 & Дания, Рибе & 0,06 & {$[1]$} \\
\hline Лопатин А.С., 2003 & Россия, Москва & 1,02 & {$[46]$} \\
\hline Johansson L., 2003 & Швеция, Шёвде & 2,7 & {$[1]$} \\
\hline Klossek J.M., 2005 & Франция & 2,1 & {$[1]$} \\
\hline Cho Y.S., 2010 & Южная Корея & 2,53 & {$[47]$} \\
\hline We J., 2015 & Южная Корея & 2,5 & {$[48]$} \\
\hline Shi J.B., 2015 & Китай & 1,1 & {$[36]$} \\
\hline Ahn J.-C., 2016 & Южная Корея & 2,6 & {$[33]$} \\
\hline Varma B.R.V., 2017 & Индия & 42,4 & {$[40]$} \\
\hline
\end{tabular}

Проведенное нами исследование свидетельствует о том, что проблема ХРС на сегодняшний день является актуальной для отечественного здравоохранения и здравоохранения зарубежных стран и далека от разрешения. Проблема ХРС с полипами также не теряет своей актуальности и все чаще поднимается в последние годы.

\section{Заключение}

Хронический риносинусит - распространенная патология ЛОР-органов среди взрослого населения, со средней частотой встречаемости в мире $11,61 \pm 5,47 \%$. В последние годы отмечается рост рассматриваемой патологии в различных странах, что требует пересмотра организационно-методического подхода к профилактике, диагностике и лечению XPC $[49 ; 50]$.

\section{Список литературы}

1. Fokkens W.J., Lund V.J., Mullol J., Bachert C., Alobid I., Baroody F., Cervin C., Cohen N., Douglas R., Georgalas C., Gevaert P., Goossens H., Harvey R., Hellings P., Hopkins C., Jones N., Joos G., Kalogjera L. Kern B., Kowalski M., Price D., Riechelmann H., Schlosser R., Senior B., Thomas M., Toskala E., Voegels R., Wang D.Y., Wormald P.J. et al. European Position Paper on Rhinosinusitis and Nasal Polyps 2012. Rhinol Suppl. 2012. Vol.23. No 3. P. 1-298.

2. Лопатин А.С., Варвянская А.В. Острый и хронический риносинусит: принципы терапии // Медицинский совет. 2014. № 3. С. 24-27.

3. Янов Ю.К., Рязанцев С.В., Страчунский Л.С., Стецюк О.У., Каманин Е.И., 
Тарасов А.А., Отвагин И.В., Крюков А.И., Богомильский М.Р. Практические рекомендации по антибактериальной терапии синусита. Пособие для врачей // Клиническая микробиология и антимикробная химиотерапия. 2003. Т. 5. № 2. С. 167-174.

4. Лопатин А.С., Гамов В.П. Острый и хронический риносинусит. М.: МИА. 2011. 46 с.

5. Крюков А.И., Студеный М.Е., Артемьев М.Е., Чумаков П.Л., Рынков Д.А., Горин Д.С. Лечение пациентов с риносинуситами: возможности консервативного и оперативного воздействия // Медицинский совет. 2012. №. 11. С. 92-96 .

6. Туровский А.Б. Хронический синусит. Новые возможности медикаментозной терапии // Поликлиника. 2013. №. 2-1. С. 66-68.

7. Иванченко О.А., Лопатин А.С. Эпидемиология хронического риносинусита: результаты многоцентрового опроса взрослой популяции // Российская ринология. 2013. Т. 21. № 3. C. 16-19.

8. Пальчун В.Т., Крюков А.И., Магомедова М.М. Руководство по очаговой инфекции в оториноларингологии. М.: ГЭОТАР-Медиа. 2015. 288 с.

9. Кривопалов А.А., Янов Ю.К., Корнеенков А.А., Астащенко С.В., Щербук А.Ю., Артюшкин С.А., Вахрушев С.Г., Тузиков Н.А. Демографические и клиникоэпидемиологические аспекты ото- и риносинусогенных внутричерепных осложнений в Российской Федерации // Инфекции в хирургии. 2015. Т. 13. № 3. С. 9-24.

10. Крюков А.И., Царапкин Г.Ю., Романенко С.Г., Товмасян А.С., Панасов С.А. Распространенность и структура заболеваний носа и околоносовых пазух среди взрослого населения мегаполиса // Российская ринология. 2017. Т. 25. № 1. С. 3-6. DOI: 10.17116/rosrino20172513-6.

11. Blackwell D.L., Collins J.G., Coles R. Summary health statistics for US adults: National Health Interview Survey 1997. National Center for Health Statistics. Vital. Health. Stat. 2002. Vol. 205. No 10. P. 1-117.

12. Collins J.G. Prevalence of selected chronic conditions: United States, 1990-1992. Vital. Health. Stat 10. 1997. Vol. 194. No 10. P. 1-98.

13. Shashy R.G., Moore E.J., Weaver A. Prevalence of the chronic sinusitis diagnosis in Olmsted County, Minnesota. Arch. Otolaryngol. Head. Neck. Surg. 2004. Vol. 130. No 3. P. 320-3. DOI:10.1001/archotol.130.3.320.

14. Soler Z.M., Mace J.C., Litvack J.R., Smith T.L. Chronic rhinosinusitis, race, and ethnicity. Am. J. Rhinol. Allergy. 2012. Vol. 26. No 2. P. 110-116. DOI:10.2500/ajra.2012.26.3741.

15. Blackwell D.L., Lucas J.W., Clarke T.C. Summary health statistics for U.S. adults: national health interview survey, 2012.Vital. Health. Stat. 2014. Vol. 260. No 10. P. 1-161. 
16. Bhattacharyya N. Incremental Health Care Utilization and Expenditures for Chronic Rhinosinusitis in the United States. Annals of Otology, Rhinology \& Laryngology. 2011. Vol. 120. No 7. P. 423-427. DOI:10.1177/000348941112000701.

17. Bachert C., Zhang L., Gevaert P. Current and future treatment options for adult chronic rhinosinusitis: Focus on nasal polyposis. Journal of Allergy and Clinical Immunology. 2015. Vol. 136. No 6. P. 1431-1440. DOI:10.1016/j.jaci.2015.10.010.

18. Hirsch A.G., Stewart W.F., Sundaresan A.S., Young A.J., Kennedy T.L., Greene S.J., Feng W., Tan B.K., Schleimer R.P., Kern R.C., Lidder A., Schwartz B.S. Nasal and sinus symptoms and chronic rhinosinusitis in a population-based sample. Allergy. 2016. Vol. 72. No 2. P. 274281. DOI:10.1111/all.13042.

19. Bhattacharyya N., Gilani S. Prevalence of Potential Adult Chronic Rhinosinusitis Symptoms in the United States. Otolaryngology-Head and Neck Surgery. 2018. Vol. 159. No 3. P. 522-525. DOI:10.1177/0194599818774006.

20. Chen Y., Dales R., Lin M. The epidemiology of chronic rhinosinusitis in Canadians. Laryngoscope. 2003. Vol. 113. No 7. P. 1199-11205. DOI:10.1097/00005537-200307000-00016.

21. Xu Y., Quan H., Faris P., Garies S., Liu M., Bird C., Kukec E., Dean S. Rudmik L. Prevalence and Incidence of Diagnosed Chronic Rhinosinusitis in Alberta, Canada. JAMA Otolaryngology-Head \& Neck Surgery. 2016. Vol. 142. No 11. P. e1-e7. DOI:10.1001/jamaoto.2016.2227.

22. Ahsan S.F., Jumans S., Nunez D.A. Chronic rhinosinusitis: a comparative study of disease occurrence in North of Scotland and Southern Caribbean otolaryngology outpatient clinics over a two month period. Scott. Med. J. 2004. Vol. 49. No 4. P. 130-133. DOI:10.1177/003693300404900404.

23. Pilan R.R., Pinna F., Bezerra T.F.P., Renata Lopes Mori R.L., Voegels R. Prevalence of Chronic Rhinosinusitis in São Paulo. Rhinology. 2012. Vol. 50. No 2. P. 129-138. DOI:10.4193/Rhino11.256.

24. Gordts F., Clement P.A.R., Buisseret T. Prevalence of sinusitis signs in a non-ENT population. Otorhinolaryngology. 1996. Vol. 58. No 6. P. 315-319.

25. Сакович А. Р. Синуситы: клинико-эпидемиологический анализ // Военная медицина. 2009. № 3. C. 60-62.

26. Hastan D., Fokkens W.J., Bachert C., Newson R.B., Bislimovska J., Bockelbrink A., Bousquet P.J., Brozek G., Bruno A., Dahlén S.E., Forsberg B., Gunnbjörnsdóttir M., Kasper L., Krämer U., Kowalski M.L., Lange B., Lundbäck B., Salagean E., Todo-Bom A., Tomassen P., Toskala E., van Drunen C.M., Bousquet J., Zuberbier T., Jarvis D., Burney P. Chronic 
rhinosinusitis in Europe--an underestimated disease. A GA(2)LEN study. Allergy. 2011. Vol. 66. No 9. P.1216-1223. DOI: 10.1111/j.1398-9995.2011.02646.x.

27. Tomassen P., Newson R.B., Hoffmans R., Lötvall J., Cardell L.O., Gunnbjörnsdóttir M., Thilsing T., Matricardi P., Krämer U., Makowska J. S., Brozek G., Gjomarkaj M., Howarth P., Loureiro C., Toskala E., Fokkens W., Bachert C., Burney P., Jarvis D. Reliability of EP3OS symptom criteria and nasal endoscopy in the assessment of chronic rhinosinusitis - a GA2LEN study. Allergy. 2010. Vol. 66. No 4. P. 556-561. DOI:10.1111/j.1398-9995.2010.02503.x.

28. Thilsing T., Rasmussen J., Lange B., Kjeldsen A.D., Al-Kalemji A., Baelum J. Chronic rhinosinusitis and occupational risk factors among 20- to 75-year-old Danes-A GA(2) LEN-based study. Am. J. Ind. Med. 2012. Vol. 55. P. 1037-1043. DOI: 10.1002/ajim.22074.

29. Hoffmans R., Wagemakers A., van Drunen C., Hellings P., Fokkens W. Acute and chronic rhinosinusitis and allergic rhinitis in relation to comorbidity, ethnicity and environment. PLoS One. 2018. Vol. 13. No. 2 P. e1- e14. DOI:10.1371/journal.pone.0192330.

30. Min Y.G., Jung H.W., Kim H.S., Park S.K., Yoo K.Y. Prevalence and risk factors of chronic sinusitis in Korea: results of a nationwide survey. Eur. Arch. Otorhinolaryngol. 1996. Vol. 253. No 7. P. 435-439.

31. Kim Y.S., Kim N.H., Seong S.Y., Kim K.R., Lee G.-B., Kim K.S. Prevalence and Risk Factors of Chronic Rhinosinusitis in Korea. American Journal of Rhinology \& Allergy. 2011. Vol. 25. No 3. P. e117-e121. DOI:10.2500/ajra.2011.25.3630.

32. Cho Y.S., Choi S.H., Park K.H., Park H.J., Kim J.W., Moon I.J., Rhee C.S., Kim K.S., Sun D.I., Lee S.H., Koo J.W., Koh Y.W., Lee K.H., Lee S.W., Oh K.W., Pyo E.Y., Lee A., Kim Y.T., Lee C.H. Prevalence of otolaryngologic diseases in South Korea: data from the Korea national health and nutrition examination survey 2008. Clin. Exp. Otorhinolaryngol. 2010. Vol. 3. No 4. P. 183193. DOI: $10.3342 /$ ceo.2010.3.4.183.

33. Ahn J.-C., Kim J.-W., Lee C.H., Rhee C.-S. Prevalence and Risk Factors of Chronic Rhinosinusitus, Allergic Rhinitis, and Nasal Septal Deviation. JAMA Otolaryngology-Head \& Neck Surgery. 2016. Vol. 142. No 2. P. E1-E6. DOI:10.1001/jamaoto.2015.3142.

34. Kim J.H., Cho C., Lee E.J., Suh Y.S, Choi B.I., Kim K.S. Prevalence and risk factors of chronic rhinosinusitis in South Korea according to diagnostic criteria Rhinology. 2016. Vol. 54. No 4. P. 329-335. DOI: 10.4193/Rhin15.157.

35. Hwang C.S., Lee H.S., Kim S.N., Kim J.H., Park D.-J., Kim K.-S. Prevalence and Risk Factors of Chronic Rhinosinusitis in the Elderly Population of Korea. American Journal of Rhinology \& Allergy. 2018. 0-0. P. 1-7. DOI:10.1177/1945892418813822. 
36. Shi J.B., Fu Q.L., Zhang H., Cheng L., Wang Y.J., Zhu D.D., Lv W., Liu S.X., Li P.Z., Ou C.Q., Xu G. Epidemiology of chronic rhinosinusitis: results from a cross-sectional survey in seven Chinese cities. Allergy. 2015. Vol. 70. No 5. P. 533-539. DOI: 10.1111/all.12577.

37. Fu Q.-L., Ma J.-X., Ou C.-Q., Guo C., Shen S.-Q., Xu G., Shi J. Influence of Self-Reported Chronic Rhinosinusitis on Health-Related Quality of Life: A Population-Based Survey. PLOS ONE. 2015. Vol. 10. No 5. P. e1- e10. DOI:10.1371/journal.pone.0126881.

38. Wang X.D., Zheng M., Lou H.F., Wang C.S., Zhang Y., Bo M.Y., Ge S.Q., Zhang N., Zhang L., Bachert C. An increased prevalence of self-reported allergic rhinitis in major Chinese cities from 2005 to 2011. Allergy. 2016. Vol. 71. No 8. P. 1170-1180. DOI:10.1111/all.12874.

39. Gao W.-X., Ou C.-Q., Fang S.-B., Sun Y.-Q., Zhang H., Cheng L., Wang Y.-J., Zhu D.-D., Lv W., Liu S.-X., Li P.Z., Xu G., Shi J., Fu Q.-L. Occupational and environmental risk factors for chronic rhinosinusitis in China: a multicentre cross-sectional study. Respiratory Research. 2016. Vol. 17. No 1. P.e1-e7. DOI:10.1186/s12931-016-0366-z.

40. Varma B.R.V., Kumar G.V. A descriptive study of diagnostic and management protocols in chronic rhinosinusitis. J. Evolution Med. Dent. Sci. 2017. Vol. 61. No 6. P. 4463-4469. DOI:10.14260/Jemds/2017/965.

41. Falliers C.J. Familial coincidence of asthma, aspirin intolerance and nasal polyposis. Ann Allergy. 1974. Vol. 32. P. 65-69.

42. Тарасов Д.И., Морозов А.Б. Частота и структура хронических заболеваний уха, горла и носа среди населения и их динамика // Вестник оториноларингол. 1991. № 2. С. 12-14.

43. Davidsson A., Hellquist H.B. The so-called "allergic" nasal polyp. ORL J. Otorhinolaryngol Relat. Spec. 1993. Vol. 55. P. 30-35. DOI: 10.1159/000276349.

44. Портенко Г.М. Распространенность полипозного синусита среди населения // Вестник оториноларингол. 1995. № 1. С. 52-54.

45. Ланцов А.А., Рязанцев С.В., Цецарский Б.М. Эпидемиология полипозных риносинуситов. СПб. РИА-АМИ. 1999. 96 с.

46. Лопатин А.С. Современные теории патогенеза полипозного риносинусита // Пульмонология. 2003. № 5. С. 110-116.

47. Cho Y.S., Choi S.H., Park K.H., Park H.J., Kim J.W., Moon I.J., Rhee C.S., Kim K.S., Sun D.I., Lee S.H., Koo J.W., Koh Y.W., Lee K.H., Lee S.W., Oh K.W., Pyo E.Y., Lee A., Kim Y.T., Lee C.H. Prevalence of otolaryngologic diseases in South Korea: data from the Korea national health and nutrition examination survey 2008. Clin. Exp. Otorhinolaryngol. 2010. Vol. 3. No 4. P. 183193. DOI: $10.3342 /$ ceo.2010.3.4.183.

48. We J., Lee W.H., Tan K.L., Wee J.H., Rhee C.-S., Lee C.H., Ahn S., Lee J.H., Kim J.-W. Prevalence of Nasal Polyps and its Risk Factors: Korean National Health and Nutrition 
Examination Survey 2009-2011. American Journal of Rhinology \& Allergy. 2015. Vol. 29. No 1. P. e24-e28. DOI:10.2500/ajra.2015.29.4131.

49. Янов Ю.К., Кривопалов А.А., Тузиков Н.А., Шнайдер Н.А., Насырова Р.Ф., Щербук А.Ю., Щербук Ю.А., Шарданов 3.Н., Артюшкин С.А.Оценка качества специализированной оториноларингологической помощи // Российская оториноларингология. 2019. Т. 18. № 1. С. 103-115.

50. Кривопалов А.А., Вахрушев С.Г. Система специализированной оториноларингологической помощи в Красноярском крае // Российская оториноларингология. 2013. №. 4. С. 50-54. 